\title{
Swing Ratios and Ensemble Timing in Jazz Performance: Evidence for a Common Rhythmic Pattern
}

\author{
ANDERS FRIBERG \& ANDREAS SUNDSTRÖM \\ Royal Institute of Technology, Stockholm
}

The timing in jazz ensemble performances was investigated in order to approach the question of what makes the music "swing." One well-known aspect of swing is that consecutive eighth notes are performed as longshort patterns. The exact duration ratio (the swing ratio) of the longshort pattern has been largely unknown. In this study, the swing ratio produced by drummers on the ride cymbal was measured. Three wellknown jazz recordings and a play-along record were used. A substantial and gradual variation of the drummers' swing ratio with respect to tempo was observed. At slow tempi, the swing ratio was as high as 3.5:1, whereas at fast tempi it reached 1:1. The often-mentioned "triple-feel," that is, a ratio of $2: 1$, was present only at a certain tempo. The absolute duration of the short note in the long-short pattern was constant at about $100 \mathrm{~ms}$ for medium to fast tempi, suggesting a practical limit on tone duration that may be due to perceptual factors. Another aspect of swing is the soloist's timing in relation to the accompaniment. For example, a soloist can be characterized as playing "behind the beat." In the second part, the swing ratio of the soloist and its relation to the cymbal accompaniment was measured from the same recordings. In slow tempi, the soloists were mostly playing their downbeats after the cymbal but were synchronized with the cymbal at the off-beats. This implied that the swing ratio of the soloist was considerably smaller than the cymbal accompaniment in slow tempi. It may give an impression of "playing behind" but at the same time keep the synchrony with the accompaniment at the off-beat positions. Finally, the possibilities of using computer tools in jazz pedagogy are discussed.

$\mathrm{O}$

NE of the most important ingredients in jazz music is the rhythm. Jazz music is supposed to "swing." One essential part of this is the rhythmic pattern sometimes called "swing eighth note pattern." It is performed by lengthening the odd eighth notes (eighth notes on the beat) and by shortening the even eighth notes (eighth notes between the beat), thus producing

Address correspondence to Anders Friberg, Dept. of Speech, Music and Hearing, Royal Institute of Technology, Drottning Kristinas v 31, SE-10044 Stockholm, Sweden. (e-mail: andersf@speech.kth.se)

ISSN: 0730-7829. Send requests for permission to reprint to Rights and Permissions, University of California Press, 2000 Center St., Ste. 303, Berkeley, CA 94704-1223. 
consecutive long-short patterns. However, the amount of lengthening and shortening is not given in the score or elsewhere, and students are often advised to learn it by listening to recordings. Gridley (1985, p. 364) notes that the pattern "falls somewhere between the tied-triplet figure and the sequence of eighth notes having identical duration." This means that the ratio (henceforth swing ratio) between consecutive eighth notes would be somewhere between 2 and 1. The same long-short pattern also occurs in many other music styles, such as classical music, folk music, and popular music. It is, for example, commonly used in French Baroque music, where it is referred to as notes inégales (Ferguson, 1975; Hefling, 1993).

Another important issue for the swing feel is the temporal relations between the different instruments in an ensemble. Improvisers often discuss the concept of playing "before the beat," "after the beat," or "on the beat." Thus, these terms define the timing relation of the improviser relative to the accompaniment, an important device for varying the musical expression (LaVerne, 1993).

Ellis (1991) let three saxophonists play a few simple melodic patterns to a computer-generated walking bass line, playing even quarter notes, together with a live piano accompaniment. They performed on a wind-controller, a MIDI device similar to a real saxophone, so that the timing could easily be extracted from the MIDI output. The swing ratios were in agreement with previous intuitive estimations, with a mean of 1.7. One of the saxophonists showed a strong dependence on tempo, with high swing ratios for slow tempi and lower ratios for faster tempi. Another saxophonist had the same swing ratio at all tempi, and the third had a dependence on tempo similar to the first, but to a lesser extent. To estimate the asynchronization, Ellis used the bass line as the timing reference and measured the saxophone delay of the downbeats. He found that all players delayed the onset relative to the bass in all cases. For the tempi 90, 120, 150,180 , and $210 \mathrm{bpm}$ (beats or quarter notes per minute), the mean delay was 40, 47, 53, 54, and $63 \mathrm{~ms}$, respectively (computed from Ellis, 1991, Table 1). Thus, there was a slight increase in delay with tempo.

Rose (1989) measured the timing of all notes played by all instruments (piano, bass, and drums) in three recordings taken from a play-along record. The compositions were examples of the jazz styles swing, jazz ballad, and latin jazz. The swing style example was played at a tempo of $132 \mathrm{bpm}$ and had a mean swing ratio of 2.4. The piano, bass, and drums all performed with approximately the same swing ratio. Rose also found that in the swing example, the drums were on average ahead of the other instruments, the piano was second, and the bass third.

Collier and Collier (1996) analyzed drummers' swing ratio at different tempi. They asked three drummers to perform the typical ride cymbal pattern (a repeated pattern of a quarter note followed by two eighth notes) at 
9 to 11 different tempi and in two conditions: swing feel and strict triple time. The drummers played on a MIDI drum pad connected to a drum machine with a cymbal sound. One of the drummers performed very consistently with a clear increase in swing ratio with decreasing tempo, whereas in the strict triple feel he closely approximated the 2:1 relation for all tempi. The other two drummers showed different patterns, both of them with a dip in the swing ratio for intermediate tempi.

The purpose of the present investigation was to investigate jazz timing further by looking at swing ratios and ensemble timing, in particular in relation to tempo. Most of the previous measurements used either laboratory recordings with a synthesized accompaniment or play-along records. It seems that these measurements generated rather mixed results, most of them with large differences for different musicians (see also Prögler, 1995). An important goal was therefore to use well-known recordings with established jazz musicians. This was made possible by making manual measurements on spectrogram displays. First, we will consider the drummer's swing ratios, second, the soloists' swing ratios and ensemble timing, and third, pedagogical applications.

\section{Drummers' Swing Ratio ${ }^{1}$}

\section{RECORDINGS}

The measurements of the drummers focused on the ride cymbal. It is often referred to as an important part that contributes to the swing feel in ensemble playing. The measurements were done on excerpts taken from commercial jazz recordings. Four drummers playing in four different jazz groups were selected (Table 1). All drummers could be considered as influential and well known, each with a distinguishable playing style within the jazz tradition. All recordings except the first were captured live. The two recordings with Tony Williams are both from the same concert at the New York Philharmonic Hall on February 12, 1964 (Carr, 1982).

\section{METHOD}

All songs considered to be in swing style with a section of ride cymbal playing were used. Most of the ballads were for this reason disregarded. One excerpt was selected from each song. These excerpts were often taken from the beginning of the first solo, that is, when the drummer started to play a regular pattern on the cymbal. In addition, four excerpts were selected from different parts of one song of each recording. This was done to see how tempo and swing ratio differed within the same song. The length of the excerpts ranged from $10 \mathrm{~s}$ to $26 \mathrm{~s}$.

\footnotetext{
1. Part of this experiment was reported in Friberg and Sundström (1997). $\quad$ [AU: add to
} ref. list\} 


\section{\{AU: Spell out tp, ts, pi in table or explain in table footnote\}}

Anders Friberg \& Andreas Sundström

TABLE 1

Recordings Used in the Analysis

\begin{tabular}{|c|c|c|c|c|}
\hline Recording & Year & Drums & Solo & Bass \\
\hline $\begin{array}{l}\text { John Coltrane } \\
\text { Aebersold Play-a-long } \\
\text { JA1244D }\end{array}$ & 1983 & Adam Nussbaum & NA & Ron Carter \\
\hline $\begin{array}{l}\text { Miles Davis } \\
\text { My Funny Valentine } \\
\text { CBS, CT9106 }\end{array}$ & 1964 & Tony Williams & $\begin{array}{l}\text { Miles Davis, tp } \\
\text { George Coleman, ts } \\
\text { Herbie Hancook, pi }\end{array}$ & Ron Carter \\
\hline $\begin{array}{l}\text { Miles Davis } \\
\text { "Four" \& More } \\
\text { CBS, CT9253 }\end{array}$ & 1964 & Tony Williams & As above & Ron Carter \\
\hline $\begin{array}{l}\text { Keith Jarrett, } \\
\text { Gary Peacock, \& } \\
\text { Jack DeJohnette } \\
\text { The Cure } \\
\text { ECM } 1440\end{array}$ & 1990 & Jack DeJohnette & Keith Jarrett, pi & Gary Peacock \\
\hline $\begin{array}{l}\text { Keith Jarrett, } \\
\text { Gary Peacock, \& } \\
\text { Jack DeJohnette } \\
\text { Standards in Norway } \\
\text { ECM } 1542\end{array}$ & 1989 & Jack DeJohnette & Keith Jarrett, pi & Gary Peacock \\
\hline $\begin{array}{l}\text { Wynton Marsalis } \\
\text { Quartet } \\
\text { Live At Blues Alley } \\
\text { CBS } 4611091\end{array}$ & 1986 & Jeff Watts & $\begin{array}{l}\text { Wynton Marsalis,tp } \\
\text { Marcus Roberts, pi }\end{array}$ & $\begin{array}{l}\text { William Robert } \\
\text { Hurst III }\end{array}$ \\
\hline
\end{tabular}

Only the soloists included in the selected excerpts are listed.

The excerpts were transferred to a PC computer, and the timing of the cymbal strokes was measured on spectrograms (see Figure 9 for an example of a typical spectrogram display). The duration from one stroke on the beat to a stroke 10 to 16 beats ahead was used to estimate the tempo of the excerpt. To estimate the swing ratio, only patterns of two consecutive eighth notes followed by a stroke on the beat were used. For each excerpt, 10 such pairs of eighth notes were collected. The swing ratio of each pair was computed as the onset-to-onset duration of the first eighth note divided by the onset-to-onset duration of the second eighth note.

The measurements were done on a PC computer with sound card SwellDSP V2.4 LSI PC/C31. The software used in the experiments was Swell Soundfile Editor v3.40.04 for recording and Gray Spectrogram Plotter v3.40 for making spectrograms. The excerpts were sampled with 16 bits resolution and with a sample frequency of $48 \mathrm{kHz}$. The fast Fourier transform analysis in the Gray software used $300 \mathrm{~Hz}$ bandwidth and a hanning window of $6 \mathrm{~ms}$. \{AU: what is hanning?\}

The strokes were measured with an estimated precision of $\pm 3 \mathrm{~ms}$. In a few cases there were two strokes very close to each other $(\sim 20 \mathrm{~ms}$ apart $)$. In these cases, the loudest stroke was chosen. A possible explanation could be that the edge of the cymbal was hit before the tip of the drumstick hit the cymbal or that the drumstick was bouncing on the cymbal. 
In some of the excerpts that were comparatively difficult to measure, the tempo measurement was repeated a few times in slightly different positions. The maximum span of these tempo measurements was $\pm 3 \mathrm{bpm}$.

\section{RESULTS}

The mean swing ratio for all excerpts is plotted in Figure 1. The general trend is an approximately linear decrease in swing ratio with increasing tempo. A few excerpts indicate an alternative approach at slow tempi with an approximately constant swing ratio of about 2 . The largest swing ratios are between 3 and 3.5. This is a larger ratio than a dotted eighth note followed by a sixteenth note. The smallest ratios approach 1 , that is, the eighth notes are played with equal duration. Note that this variation in swing ratio is not at all in agreement with the common idea that the swing ratio is close to 2 , also referred to as "triple feel." This was the case for one particular tempo only, at about $200 \mathrm{bpm}$.

Figure 2 shows the absolute duration of the short tone of the long-short pattern as a function of tempo. Interestingly, for medium to fast tempi $(>150 \mathrm{bpm})$ the duration of the second note is approximately constant at about $100 \mathrm{~ms}$. This may indicate a limit of note duration (see discussion section).

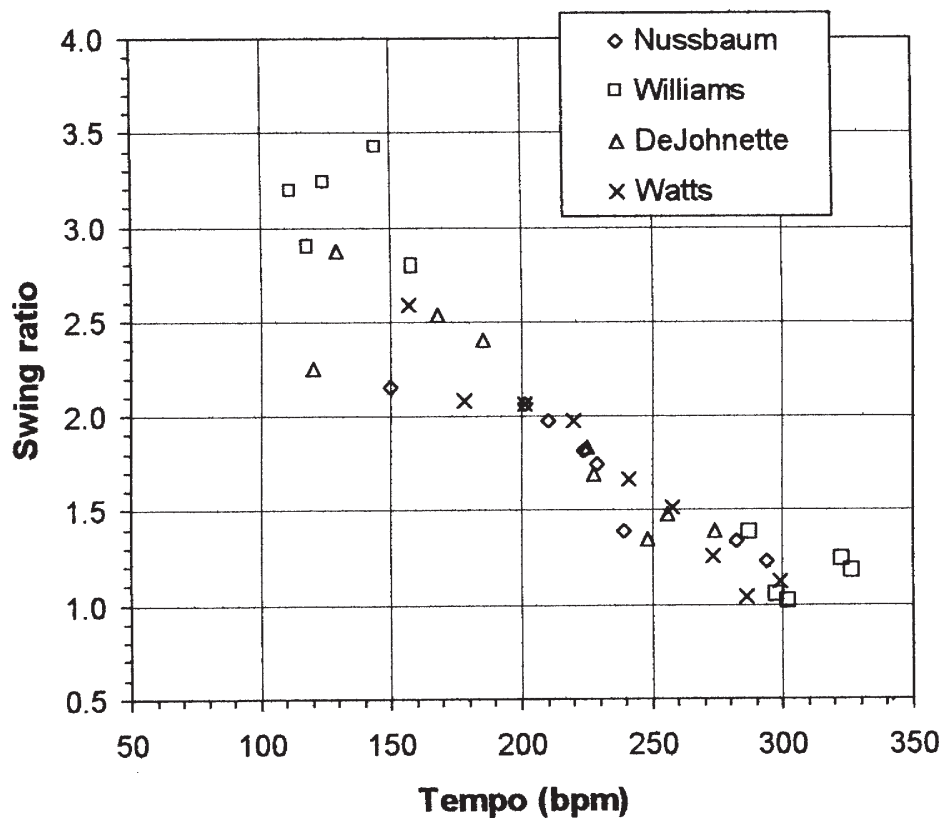

Fig. 1. Drummers' swing ratio as a function of tempo. All measurements are from the drummers' ride cymbal. 


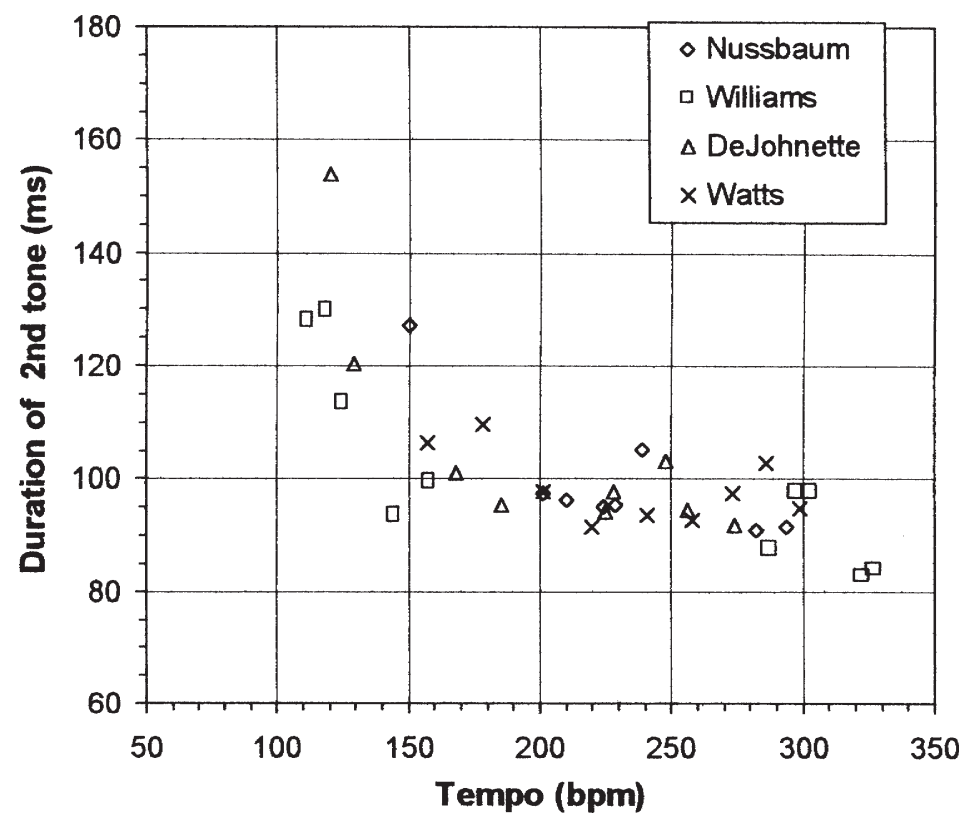

Fig. 2. The absolute duration of the second tone as a function of tempo for the four drummers and all excerpts.

The swing ratios of each drummer are separated into four graphs in Figure 3. The drummers exhibit a surprisingly consistent swing ratio in view of the large differences in drumming style. Most of the differences between the drummers are found for tempi less than $200 \mathrm{bpm}$. Williams had the highest ratio values for the slow excerpts. Watts follows the same trend but there is only one sample that goes in that direction. Similarly, the ratios for Nussbaum flatten out for slower tempi, but there is only one sample there too. DeJohnette had one clear deviation from the general trend of a linear curve that suggests a deliberate change of swing ratio within the same tempo range. The difference between drummers was confirmed in a two-way analysis of covariance with drummer as one factor (4 levels), tempo as regression variable, and with swing ratio as the dependent variable. Not surprisingly, the influence of tempo was highly significant $(F=186, \mathrm{p}<$ $.0001)$ but also the influence of drummer was significant $(F=4.4, p<.01)$. The interaction of tempo and drummer was not significant $(F=2.6, p<$ $.07)$.

Included in Figure 3 are also the standard deviations for each excerpt. These intervals show that the variation within each excerpt differed between drummers. Williams had the largest variation in his slow tempi, and Nussbaum the smallest variation within each excerpt. 

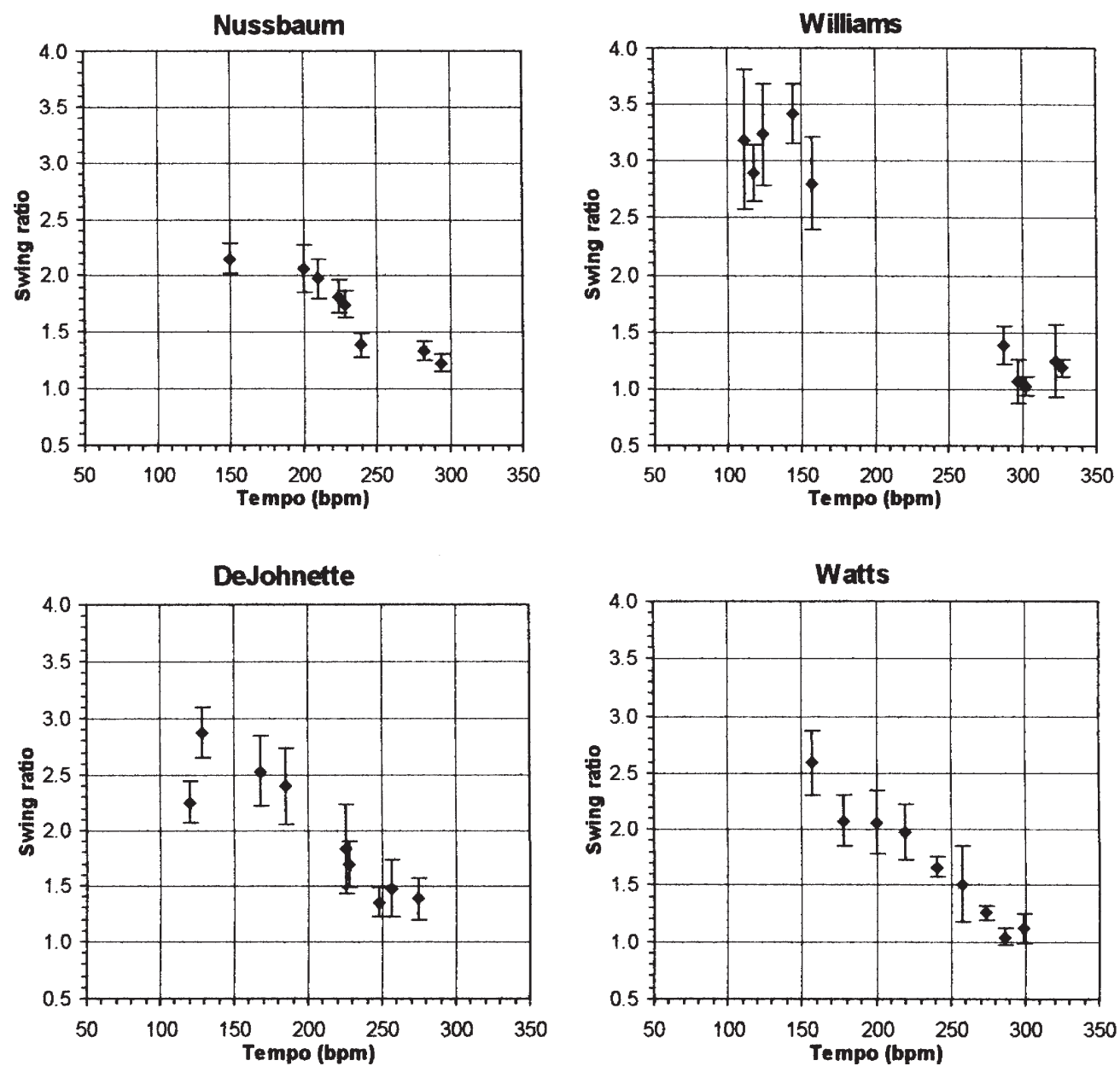

Fig. 3. Swing ratio for the four drummers as a function of tempo. Error bars show the standard deviation.

\section{VARIATIONS WITHIN THE SAME SONG}

The swing ratios in four different positions within a song are shown in Figure 4. There is very little indication of a linear change in swing ratio within each song, possibly with the exception of Nussbaum and Watts. The times between samples varied but were roughly 1 minute. The overall trend is to continuously increase the tempo during the song; there is no example of a decrease in tempo in these few examples (cf. Collier \& Collier, 1994). The difference between the first and the last tempo is in most cases quite large (Impressions: 12\%, My Funny Val.: 16\%, So What: 11\%, Woody'n.: 7\%, Delfeayo's Dil.: 3\%). 

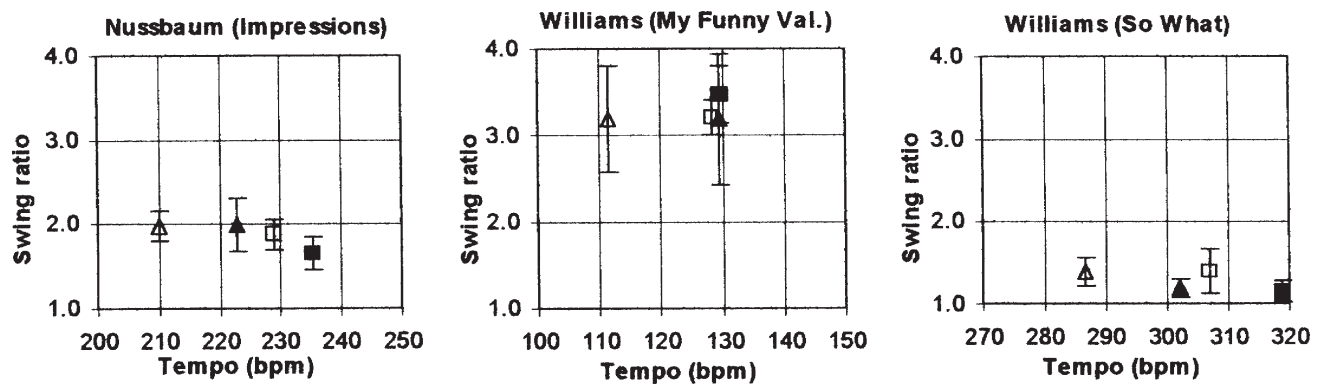

DeJohnette (Woody'n...)
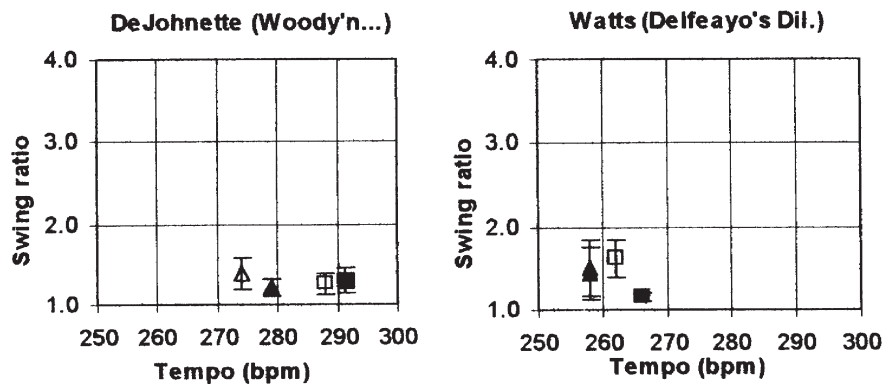

$\Delta$ Position 1

$\Delta$ Position 2

$\square$ Position 3

- Position 4

Fig. 4. Drummers' swing ratio as a function of tempo in four positions within one song.

\section{Ensemble Timing and Soloist Swing Ratio}

The second part of this article concerns the timing relations between the performers in a typical jazz ensemble, as well as estimations of the soloists' swing ratios.

\section{METHOD}

The music excerpts used were the same as those used to examine drummers' swing ratio, but the excerpts were sampled down to $24 \mathrm{kHz}$ in order to more easily observe the soloist and bass timing in the spectrograms. Twenty-one of the original 40 excerpts could be used. Excerpts were selected in which the soloist played some successive eighth notes. No examples of bass or drum solo were selected. In these 21 excerpts, all cymbal onsets, soloist onsets, and if possible bass onsets were measured on spectrograms. The swing ratios were computed from two successive interonset intervals, that is, only the cases with at least two eighth notes within a beat followed by a new note were used. Beat and off-beat delays between drummer and soloist/bass were estimated from all simultaneous tones within the same sequence.

Naturally, the number of estimated points varied greatly between excerpts because of differences in playing. Excerpts containing fewer than three estimated points were omitted. For the swing ratio measurement, two excerpts were omitted, yielding a mean number of estimated points equal to 11 . For the beat measurements, the minimum number of estimated points was 5 , with a mean of 18 . For the off-beat measurements, four excerpts were omitted, resulting in a mean number of estimated points equal to 6 . The delay of the bass could be estimated in 13 excerpts. The lowest number of estimated points was 5 and the mean was 19 points. 


\section{RESULTS}

The swing ratios of the soloists and drummers within the selected sequences are shown in Figure 5. For medium tempi, it is clear that the soloists' swing ratios are considerably lower than the drummers' swing ratios and that all means are below 2, that is, they are all more even than a strict triple feel. Interestingly, this finding is in agreement with the previous statement that the swing ratio is between 1 and 2 (Gridley, 1985; LaVerne, 1993). Also, it shows that it is important to distinguish between different instruments and function (solo/accompaniment) when discussing swing ratios.

Given the small number of measurements and the rather large variation within each excerpt and between different musical materials, it is not meaningful to make conclusions about each musician's timing from the data in Figure 5. To do so, it would be necessary to do a controlled experiment in which the soloist plays the same material, such as reported in Ellis (1991), or to use a large data set.

The mean delay of each soloist relative to the ride cymbal is shown in Figure 6 for the quarter notes on the beat. Note that in only 1 out of 21 cases was the soloist playing before the ride cymbal (negative mean value in the figure). None of these occur for tempi below $250 \mathrm{bpm}$. Despite all

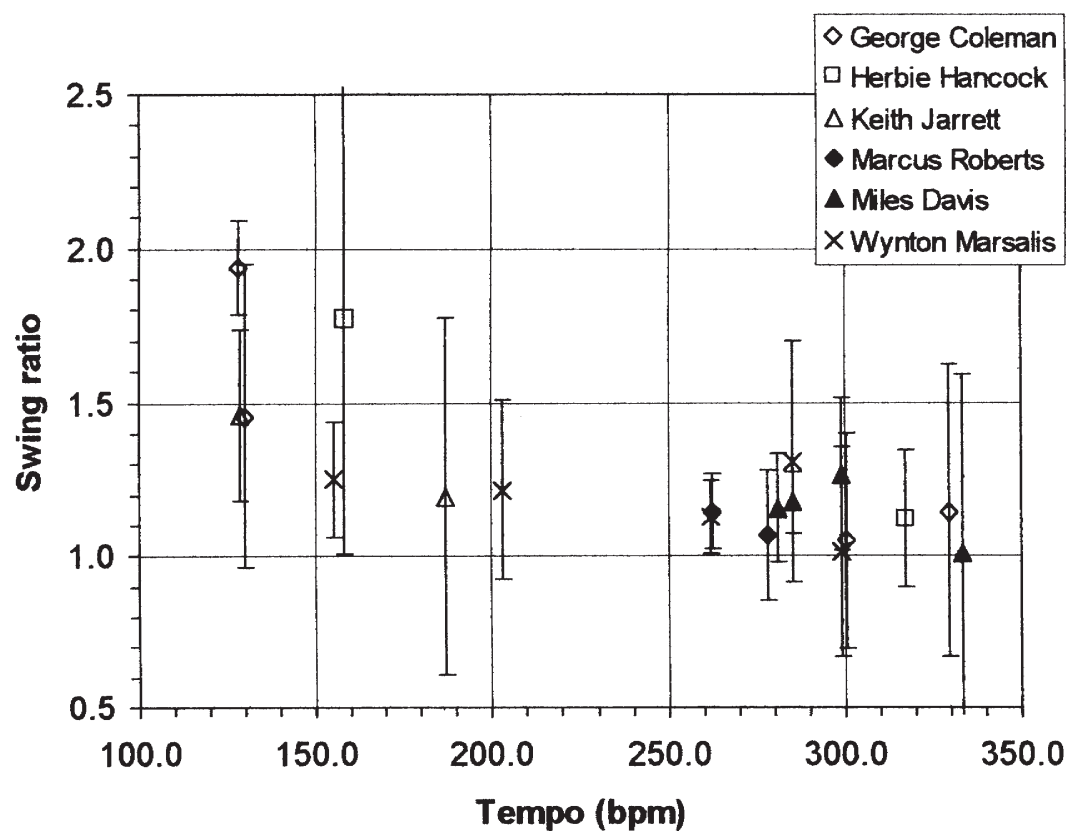

Fig. 5. Soloists' swing ratio as a function of tempo. The error bars show the standard deviation. 


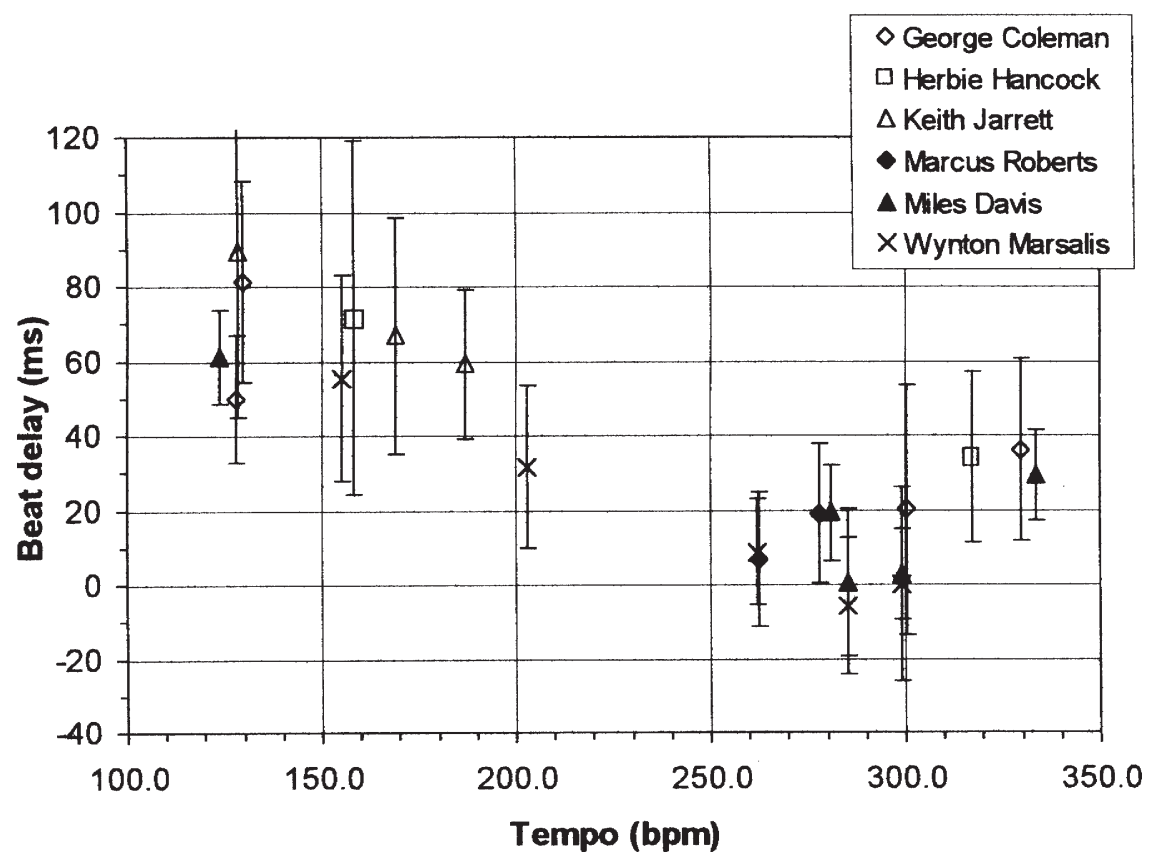

Fig. 6. Soloists' downbeat delay as a function of tempo. The error bars show the standard deviation.

discussions about playing before or after the beat, the data shows that all soloists' in general are playing after the beat (or at least after the ride cymbal). This finding was also in agreement with Ellis' (1991) findings. A linear trend can be observed with more delay for slower tempi. This result was, however, opposite to the finding by Ellis of a slight increase in the delay with tempo. Again, it is hard to observe any individual differences in the limited data set.

The corresponding delay for the off-beats is shown in Figure 7. Interestingly, a different pattern emerges with no tendency to play either before or after the ride cymbal. Rather, a remarkable synchronization at the off-beats is apparent. The standard deviations are also smaller than for the downbeats. The rigid placement of these tones may have two possible explanations: (1) Only the downbeats that are used as the expressive device altering the timing "feel," or (2) the delay range is smaller for the off-beat tones because a new ride cymbal strike is obtained only about $100 \mathrm{~ms}$ later. The only exception is the three very fast excerpts, where all the notes seem to be delayed relative to the ride cymbal.

The delay of the bass relative to the ride cymbal is shown in Figure 8. In most of the excerpts, the bass is slightly delayed. There may be a tendency for longer delays at slower tempi. 


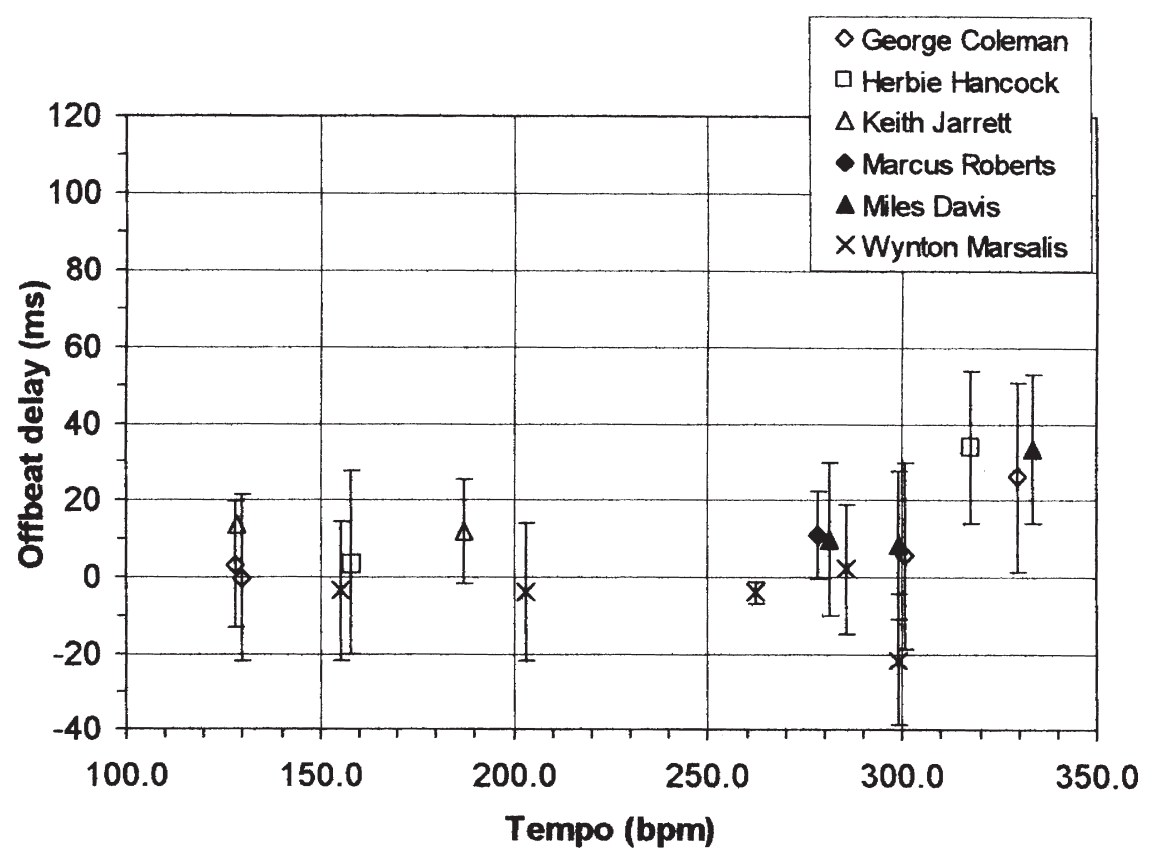

Fig. 7. Soloists' off-beat delay as a function of tempo. The error bars show the standard deviation.

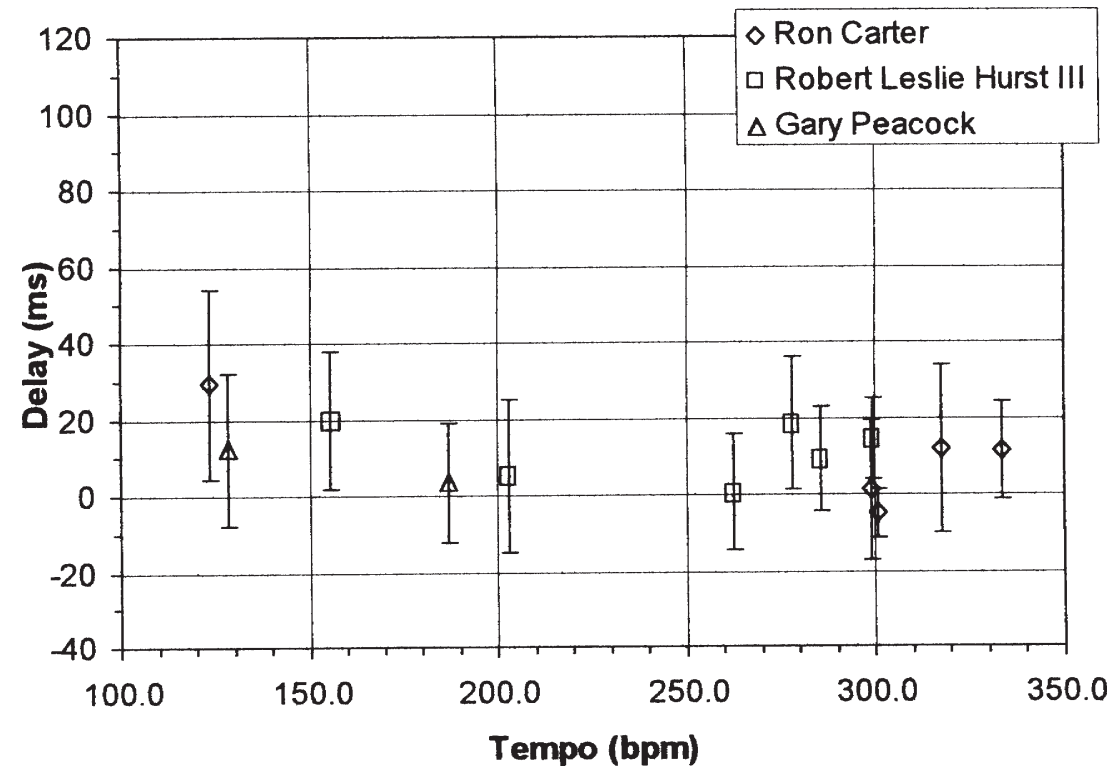

Fig. 8. Delay of the bass relative to the ride cymbal as a function of tempo. The error bars show the standard deviation. All measurements are from downbeat positions. 
To summarize, the interaction of the musicians in a jazz ensemble can be characterized in the following way. The drummer plays with a rather large swing ratio, with values up to 4 for slow tempi. The soloist has a smaller swing ratio, typically between 1 and 2 . This difference is possible because the soloist is delayed at the downbeats and synchronized at the off-beats. This intricate timing interplay between the musicians is presumably an important component in generating a good swing feel. An example is given in Figure 9, which is taken from a spectrogram from one of the analyzed excerpts.

\section{Pedagogical Applications}

Contrary to tonal and harmonic exercises, few attempts have been made to define exercises for developing the sense of jazz timing. At the same time, most pedagogues and musicians claim that good timing is the most

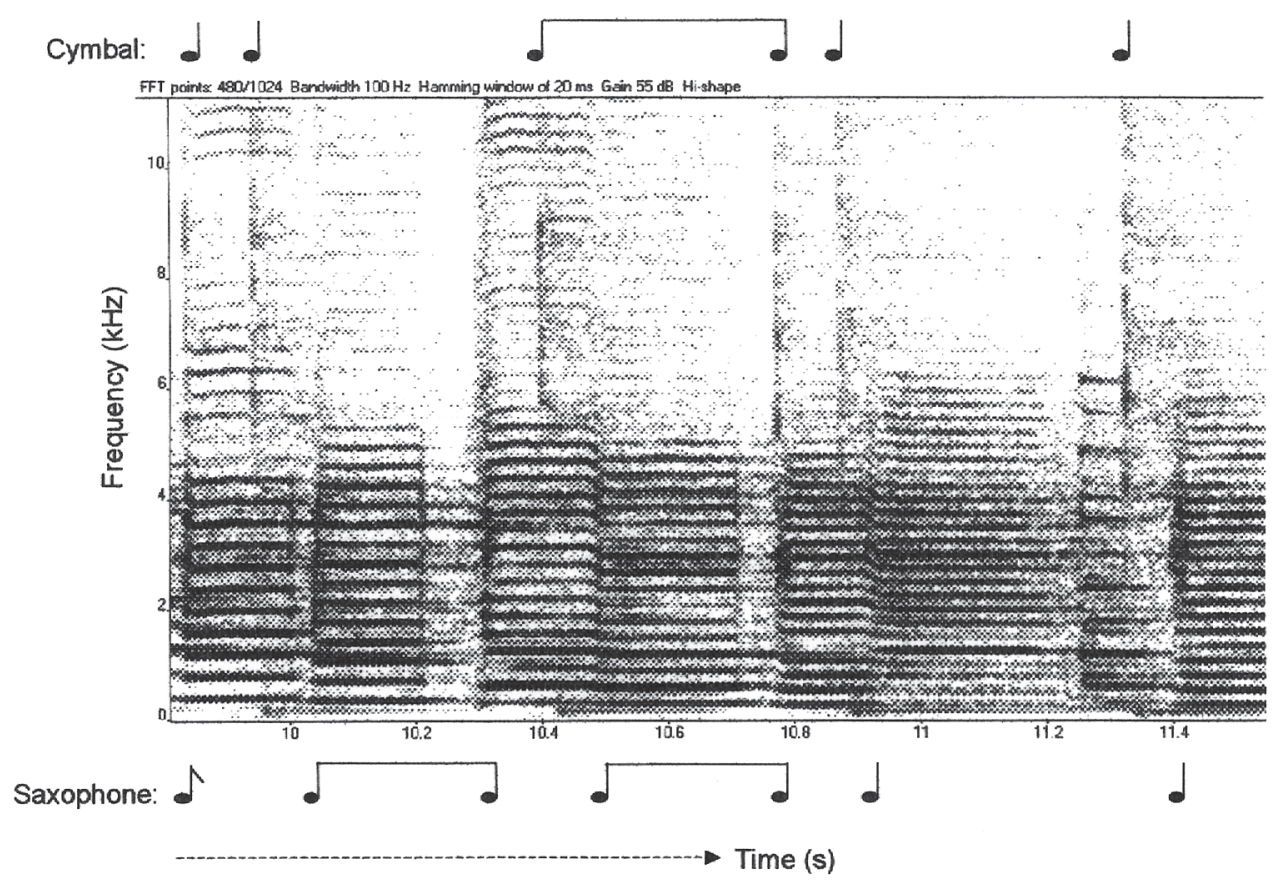

Fig. 9. A spectrogram illustrating a typical timing pattern of a jazz ensemble playing medium tempo swing. The excerpt is from "My Funny Valentine" performed by Miles Davis Quintet. The cymbal onsets appear as vertical lines in the higher part of the graph. The saxophone onsets appear as breaks in the horizontal lines in the lower part of the graph. The lowest horizontal lines are from the bass. In this example, the cymbal is being played with a swing ratio of about 4 and the saxophone with a swing ratio of approximately 3:2. The downbeat saxophone tones are delayed relative to the cymbal by about $100 \mathrm{~ms}$, but on the off-beats, the cymbal and the saxophone are synchronized. 
important factor in jazz playing. When timing exercises are suggested, there are often simplifications that can lead to undesirable results. For example, even in some recent instructions in jazz drumming, the advice is to play exactly triplets (e.g., De Simone, 2000). As we have seen, exact triplets seem to occur only at one particular tempo. Apparently, only some musicians are aware of the change in swing ratio with tempo. Without this knowledge, it is hard for a teacher to give constructive advice about the swing feel to a beginning jazz player.

The present findings indicate that there is a basic pattern that the included musicians followed more or less closely. It might be advantageous to try to learn that pattern and make conscious deviations from it in order to change the feel. For example, a common pitfall among jazz soloists is to play with too much swing ratio (LaVerne, 1993). This is natural because most jazz drummers use high swing ratios. However, if the student practices delaying the downbeats and synchronizing the off-beats, a more even swing pattern will occur automatically. As accompaniment, one can use a drum machine programmed with the proper swing ratio according to current tempo or a play-along record. To use specific feedback of the physical parameters, such as the timing, can have a large effect on the development of musical expression (Juslin \& Laukka, 2000). Here, the feedback can be improved by using a normal spectrogram program that displays the timing relations and swing ratios, similarly to how the present data were collected. Recently, such programs, including real-time analysis, have become available free on the Internet (e.g., Sjölander \& Beskow, 2001). Thus, the only equipment needed is a personal computer and a microphone.

\section{Discussion}

In classical music, the melody part is often played earlier than the accompaniment. In measurements of string and wind trios as well as in piano performances, the melody is typically played about $20 \mathrm{~ms}$ before the other voices (Goebl, 2001; Palmer, 1996; Rasch, 1979). It will have the effect of emphasizing the melody because if two notes are presented at almost the same time, the first note will be perceptually emphasized (Rasch, 1978). In jazz at slow to medium tempi, the melody is instead mostly played after the accompaniment. The purpose here is probably not to highlight the soloist; most of the time the soloist is easily recognized because of either large spectral differences or the use of microphones. One possible interpretation is that delayed downbeats and synchronized off-beats may create both the impression of the "laid-back" soloist, which is often strived for in jazz, and at the same time an impression of good synchronization.

This delay of the soloists' downbeats and the synchronized off-beats indicate that the concept of "playing behind" means that only the downbeats are played late. That is, different playing styles might be obtained by 
changing the timing pattern rather than playing the whole pattern early or late. This is also supported by the present data, which show the standard deviation is larger for the downbeats (Figure 6) than for the off-beat tones (Figure 7), implying that the expressive variation is found in the placement of the downbeats. A similar case was mentioned by drummer Peter Erskine in a workshop in Stockholm in 1997. There is a typical jazz accompaniment pattern in which one hand plays even quarter notes on the ride cymbal and the other hand a rim shot at the fourth quarter note in the measure. Erskine found that a "behind-the-beat" playing style was obtained by only delaying the rim shot.

One aspect in evaluating these data is how large deviations are perceptible. The just noticeable difference (JND) of the swing ratio was found to be about $20 \%$ in a music example at a tempo of $170 \mathrm{bpm}$ (Friberg \& Sundberg, 1994; see also Friberg, 1995). The JND was measured in rela-

\{AU: 63 bpm or 63 patterns per minute?\} tion to even eighth notes, thus the ratio had to change from 1 to 1.2 to be noticeable. About $20 \%$ was also obtained for deviations from a 2:1 pattern at a tempo of 63 patterns per minute. These experiments used simple melodies played on a synthesizer. In psychoacoustic experiments where every other onset was delayed in repetitive sequences, thus, similar to a sequence of swing eight notes but with simple acoustic markers instead of musical tones, the JND in swing ratio was about $10 \%$ for tempi slower than 120 bpm (cyclic displacement in Friberg \& Sundberg, 1995). For tempos from 120 to $300 \mathrm{bpm}$, the JND was constant at about $10 \mathrm{~ms}$.

Interestingly, the duration of the second note (Figure 2) is also constant for a wide range of tempi. If we assume this lower JND of $10 \mathrm{~ms}$ and a constant second-note duration of $100 \mathrm{~ms}$, we get the JND in swing ratio as shown by the error bars in Figure 10. In terms of percentages of the swing ratio, the JND will be about $10 \%$ at $120 \mathrm{bpm}$ and about $20 \%$ at $300 \mathrm{bpm}$. These values could serve to give an idea of the JND quantity. However, for a reliable estimation of the JND, it is necessary to measure it for several tempi and swing ratios.

A direct implication of a tempo-dependent swing ratio is that a swing performance is not invariant under tempo transposition, that is, it cannot be transposed in tempo by multiplying all durations with a constant factor. Such nonproportional scaling of timing patterns was also found by Desain and Honing (1994) when they measured performances of the same piece at three different tempi. They used a theme and a variation by Beethoven (WoO 70) that contained many repetitive metrical patterns including a 2:1 pattern of a quarter note followed by an eighth note, thus, similar to the swing type performance of long-short patterns. Repp (1994), on the other hand, found that performances of Schumann's Träumere $i$ were largely invariant under tempo transposition. Träumerei has, however, an entirely different character with considerably longer tones and with no repetitive 


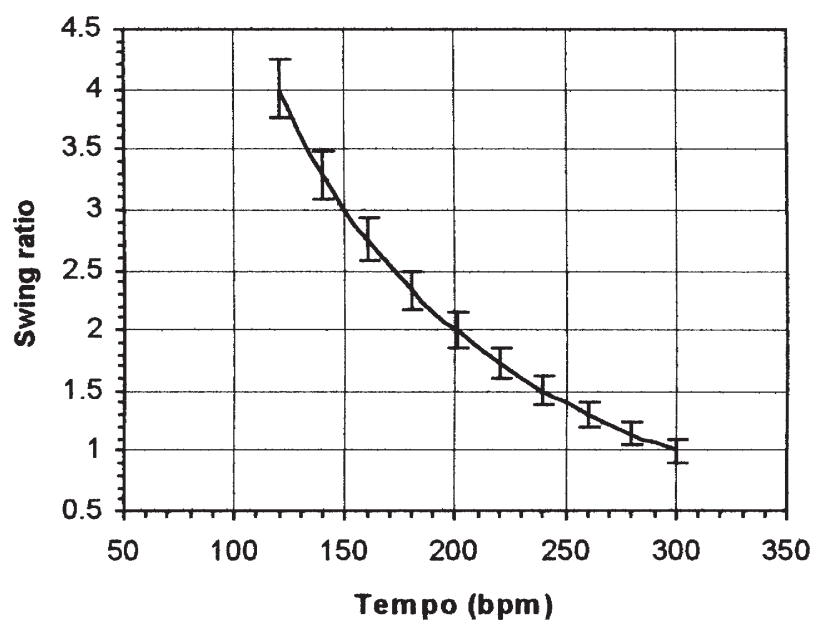

Fig. 10. The curve shows the swing ratio assuming a constant second-note duration of 100 ms. The error bars shows the just-noticeable difference (JND) in swing ratio as a function of tempo, assuming a constant JND in duration of $10 \mathrm{~ms}$.

metrical patterns. The tone interonsets in Träumerei were all longer than $250 \mathrm{~ms}$, thus within the range where the JNDs follow Weber's law, that is, are constant percentages of the interonset intervals (Friberg \& Sundberg, 1995).

In Figure 2, the duration of the short note reaches a limit around 80 to $100 \mathrm{~ms}$, which is similar to the shortest eighth notes used for a melodic line in jazz. The upper tempo limit in jazz is around 320 bpm (Collier \& Collier, 1994), which corresponds to an eighth-note duration of $94 \mathrm{~ms}$. It is also similar to the limit of the perceptual duration of a short stimulus found by Efron (1973). He found that the shortest perceptual duration was about $130 \mathrm{~ms}$, regardless of modality (auditory, visual, or tactile), meaning that the perceptual experience of a short pulse persists for that amount of time even if the physical duration is shorter. This effect has been used to explain a number of visual phenomena such as flicker fusion. This limit on perceptual duration would then imply that sequences of tones with shorter duration would blend into each other, making it hard to distinguish each tone in a melody. Rather, the melodic line will be perceived as a shape or "color." Interestingly, when John Coltrane started to play very fast runs on the saxophone in the late 1950s, which was something new for that time, it was described as "sheets of sound."

There were quite large standard deviations for the soloists' swing ratios, soloists' downbeat delay, and some of the drummers' swing ratios. The relatively small variations in, for example, off-beat delay, provide some evidence that the large variations are a result of expressive variation rather 
than playing inaccuracies. In comparing drummers, it can be noted that Williams had large variations in swing ratio, particularly in the slow excerpts, whereas Nussbaum had considerably lower variations (Figure 3). Williams was interacting with a soloist playing different rhythmic material, whereas Nussbaum just played a straight accompaniment. One possibility for a drummer to change swing ratio would be to synchronize with different subdivisions by the soloist. When the soloist is playing triplets or sixteenth notes, a drummer can synchronize to those patterns playing a ratio of 2 or a ratio of 3 , respectively.

This study did not reveal the characteristics of each musician's personal time feel. Rather, it showed, rather surprisingly, that these musicians shared a common pattern of timing. We believe, however (as most musicians already know), that each musician has a preferred timing characteristic. Because the variation in the measurements was quite large, a substantially larger collection of data set is needed to determine this.

\section{Conclusion}

This study showed that musicians in a jazz ensemble have an intricate common pattern of timing related to which instrument is played and to the tempo. In particular, the following points could be mentioned: (1) The commonly mentioned "triple-feel," that is, a swing ratio of 2 , was not favored in the measurements. Rather the swing ratio varied linearly with the tempo. (2) The drummers had a rather high swing ratio at slow tempi, even exceeding a dotted eighth followed by a sixteenth note, whereas the soloists had small swing ratio, usually less than 2 at medium tempi. (3) The soloist were in most cases playing after the drums on the downbeat and synchronized with the drums on the off-beats. Surprisingly, these findings indicated in some cases a rather large discrepancy between common beliefs and "the physical reality," as in the case of drummers' swing ratio, which often is believed to be close to 2 (triplet subdivision) whereas the real value sometimes exceeded 3 (sixteenth subdivision). ${ }^{2}$

\section{References}

Carr, I. (1982). Miles Davis, A Critical Biography. London: Paladin Grafton Books.

Collier, G. L., \& Collier, J. L. (1994). An exploration of the use of tempo in jazz. Music Perception, 11, 219-242.

Collier, G. L., \& Collier, J. L. (1996). The swing rhythm in jazz. Proceedings of the International Conference on Music Perception and Cognition, Montreal. Montreal: ICMPC.

2. We thank Patrik Juslin and Walter Gerard Busse for valuable comments on a first draft. This work was supported by a grant from the Bank of Sweden Tercentenary Foundation.

\{AU: Page range for Collier \& Collier? Publisher and city of publication $\mathrm{OK}$ ?\} 
De Simone, M. (2000). Learning to play jazz, Part 1. Modern Drummer, May, 102-103.

Desain, P., \& Honing, H. (1994). Does expressive timing in music performance scale proportionally with tempo? Psychological Research, 56, 285-292.

Efron, R. (1973). An invariant characteristic of perceptual systems in the time domain. In S. Kornblum (Ed.), Attention and Performance IV (pp. 713-736). New York: Academic Press.

Ellis, M. C. (1991). An analysis of "swing" subdivision and asynchronization in three jazz saxophonists. Perceptual and Motor Skills, 75, 707-713.

Ferguson, H. (1975). Keyboard interpretation. New York: Oxford University Press.

Friberg, A., \& Sundberg, J. (1994). Just noticeable difference in duration, pitch and sound level in a musical context. In I. Deliège (Ed.), Proceedings of $3 \mathrm{rd}$ International Conference on Music Perception and Cognition, Liège (pp. 339-340). Liège: ICPMC. \{AU: Publisher and city OK?\}

Friberg, A., \& Sundberg, J. (1995). Time discrimination in a monotonic, isochronous sequence. Journal of the Acoustical Society of America, 98, 2524-2531.

Friberg, A. (1995). A quantitative rule system for musical performance. Doctoral dissertation, Royal Institute of Technology, Sweden.

Friberg, A., Sundberg, J., \& Frydén, L. (1994). Recent musical performance research at KTH. In J. Sundberg (Ed.), Proceedings of the Aarhus Symposium on Generative Grammars for Music Performance (pp. 7-12). \{AU: Need publisher and city of publication. Also, where is this ref. cited in text or figure or table? Please cite or delete.

Goebl, W. (2001). Melody lead in piano performance: Expressive device or artifact? Journal of the Acoustical Society of America, 110, 563-572.

Gridley, M. C. (1985). Jazz styles history and analysis. 2nd ed. CITY?: Prentice-Hall.

Hefling, S. E. (1993). Rhythmic alteration in seventeenth- and eighteenth-century music: Notes inégales and overdotting. New York: Schirmer Books.

Juslin, P. N., \& Laukka, J. (2000). Improving emotional communication in music performance through cognitive feedback. Musica Scientiae, 4, 151-183.

LaVerne, A. (1993). Six in-depth exercises in jazz time. Keyboard, July, 72-86.

Palmer, C. (1996). On the assignment of structure in music performance. Music Perception, 14, 23-56.

Prögler, J. A. (1995). Searching for swing: Participatory discrepancies in the jazz rhythm section. Ethnomusicology, 39, 21-54.

Rasch, R. A. (1978). The perception of simultaneous notes such as in polyphonic music. Acustica, 40, 21-33.

Rasch, R. A. (1979). Synchronization in performed ensemble music. Acustica, 43, 121131.

Repp, B. H. (1994). Relational invariance of expressive microstructure across global tempo changes in music performance: An exploratory study. Psychological Research, 56, 269284.

Rose, R. F. (1989). An analysis of timing in jazz rhythm section performance. PhD dissertation, University of Texas, CITY?.

Sjölander, K., \& Beskow, J. (2001). WaveSurfer. Available at http://www.speech.kth.se/ wavesurfer/ 